\title{
Direct Observation of Phase Transformations in Ge-Sb-Te Materials
}

\author{
Manish Singh $^{1}$, Chanchal Ghosh ${ }^{1}$, Paul Kotula ${ }^{2}$, John Watt ${ }^{3}$, Helena Silva ${ }^{1}$ and C Barry Carter ${ }^{4,5}$ \\ ${ }^{1}$ Department of Electrical and Computer Engineering, University of Connecticut, Storrs, Connecticut, United \\ States, ${ }^{2}$ Department of Materials Characterization, Sandia National Laboratories, Albuquerque, New Mexico, \\ United States, ${ }^{3}$ Center for Integrated Nanotechnologies, Los Alamos, New Mexico, United States, ${ }^{4}$ Center for \\ Integrated Nanotechnologies, Sandia National Laboratories, Albuquerque, New Mexico, United States, \\ ${ }^{5}$ Department of Electrical and Computer Engineering, University of Connecticut, Storrs, Connecticut, United \\ States
}

Phase-change materials (PCM) have potential application in next-generation programmable random-access memories (RAM) [1]. Germanium-Antimony-Tellurium (GST) alloy is one of the extensively studied system owing to its large change in resistance during phase transformation from amorphous to crystalline [2]. Moreover, phase changes take place at the time scale of nanoseconds require to probe the dynamics of processes at relatively higher temporal resolution. This is expected to unearth the nearly closer transformation temperatures in real time and in-situ TEM at high resolution is possibly the ideal tool for this purpose. There are several reports of studies of crystallization kinetics in GST based on thermal calorimetry, Raman spectroscopy, electron microscopic observations on ex-situ annealed films on substrates, etc. [3]. Determination of precise transformation temperatures from amorphous to crystalline phase has also been reported through in-situ TEM investigation albeit at relatively lower temporal resolution, based on electron diffraction and low magnification TEM imaging before and after crystallization [4]. Additionally, determination of crystallization temperature of phase transformation is complicated by the interfacial effects of substrates and protective layers with the GST as well as thermal gradient during heating. To overcome these issues, GST films have been directly deposited on Protochips. This strategy helps to minimize the interference from the substrate and protective layer during investigation of phase changes through TEM. The details of the in-situ methodology is well described in the available literature [5].

In this investigation $\mathrm{Ge}_{2} \mathrm{Sb}_{2} \mathrm{Te}_{5}$ thin films of thicknesses $10 \mathrm{~nm}, 20 \mathrm{~nm}$ and $30 \mathrm{~nm}$ were directly deposited on Protochips using magnetron sputtering. In-situ heating experiments has been carried out in a $\mathrm{C}_{\mathrm{s}}$-corrected FEI Titan ETEM employing Aduro 300 Protochips holder. Dynamics of phase transformations have been captured using Gatan K3-IS camera at low dose to eliminate the effect of e-beam induced knock-on damage to the sample. Protochips were heated @ $5{ }^{\circ} \mathrm{C} / \mathrm{sec}$ from room temperature to $140{ }^{\circ} \mathrm{C}$. The chemical composition of $\mathrm{Ge}_{2} \mathrm{Sb}_{2} \mathrm{Te}_{5}$ films before and after heat-treatments are analyzed using STEM-XEDS in a Thermofisher Tecnai F30.

General morphology of the films and the corresponding phase structural information have been studied for the GST films of thickness $10 \mathrm{~nm}, 20 \mathrm{~nm}$, and $30 \mathrm{~nm}$, respectively. The morphology of the as-grown films at room temperature for all thickness are observed as islands type. The diffuse rings indicating the amorphous nature of the films in as-grown condition. The HRTEM image snapshots captured during heating of GST films of thickness $20 \mathrm{~nm}$ are presented in Figure 1. Corresponding power spectrum displayed in Figure 2 show the crystallization from amorphous to crystalline phase and could be indexed to $\mathrm{Ge}_{2} \mathrm{Sb}_{2} \mathrm{Te}_{5}$ fcc. The GST thin films deposited on oxidized $\mathrm{Si}$ and $\mathrm{Si}_{3} \mathrm{~N}_{4}$ substrates have been reported to display two distinct phase transformations i.e., from amorphous to fcc and fcc to hep, respectively. In the present investigation, first sign of crystallization shows lattice fringe spacings corresponding to $\mathrm{Ge}_{2} \mathrm{Sb}_{2} \mathrm{Te}_{5}$ fcc is observed at $\sim 2 \mathrm{~s}$. This suggests that despite imaging in low-dose conditions, the effect of beam induced crystallization could not be eliminated. It has been observed that both fcc and hcp phases were found to coexist in the entire temperature range albeit with the varied phase fraction.

This research is funded by NSF under award DMR-1710468. TEM investigation was carried out at CINT, an Office of Science User Facility operated for the U.S. DOE, and in the Materials Characterization Department. 
Sandia National Labs is managed and operated by National Technology and Engineering Solutions of Sandia, LLC., a wholly owned subsidiary of Honeywell International, Inc., for the U.S. DOE's NNSA contract DENA-0003525. The views expressed here do not necessarily represent the views of the U.S. DOE or the U.S. Government. Los Alamos National Laboratory, an affirmative action equal opportunity employer, is managed by Triad National Security, LLC for the U.S. Department of Energy's NNSA, under contract 89233218CNA000001.

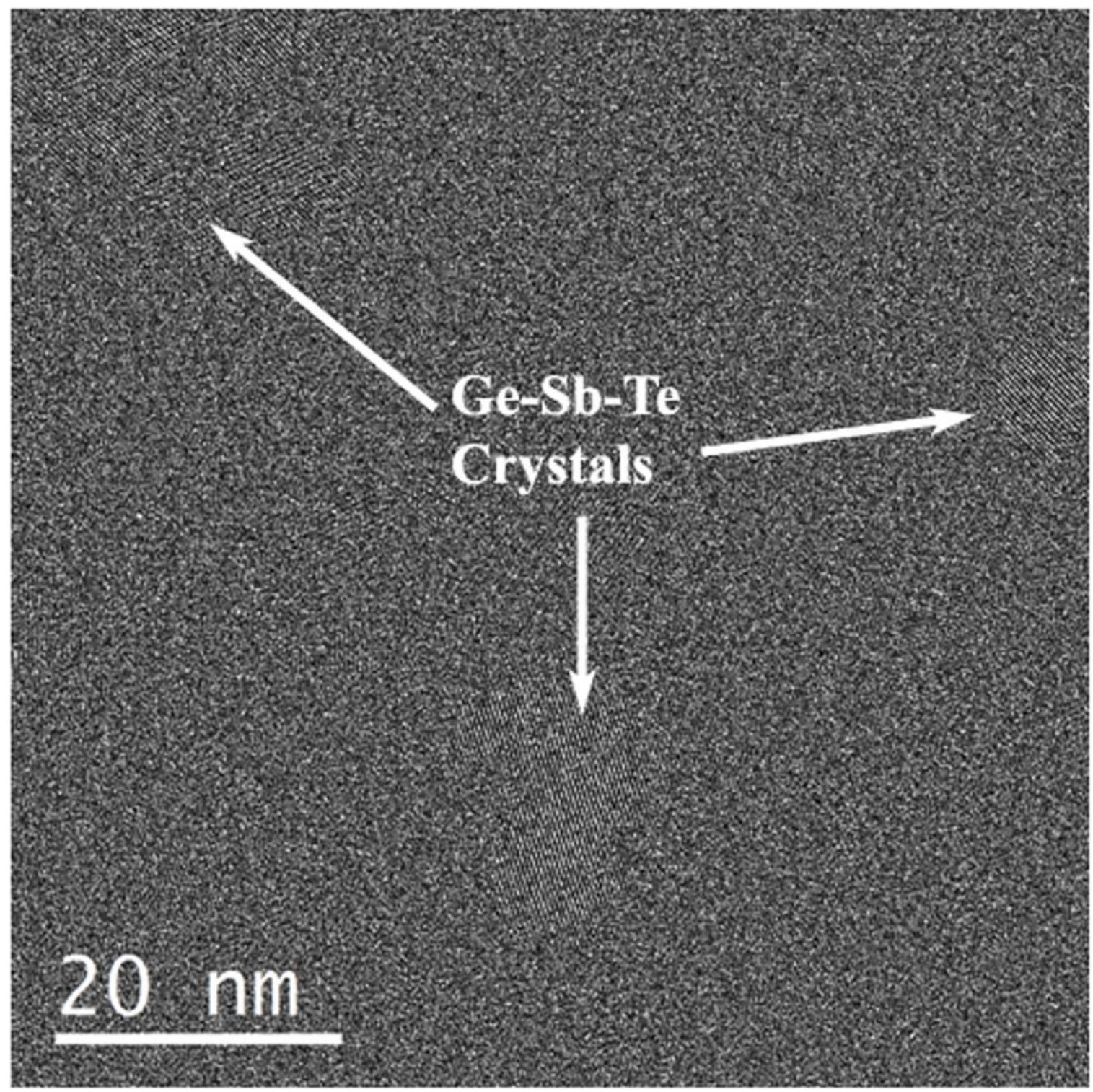

Figure 1. The Ge2Sb2Te5 crystals after phase transformation from amorphous phase and corresponding power spectrum captured in a K3-IS camera. 


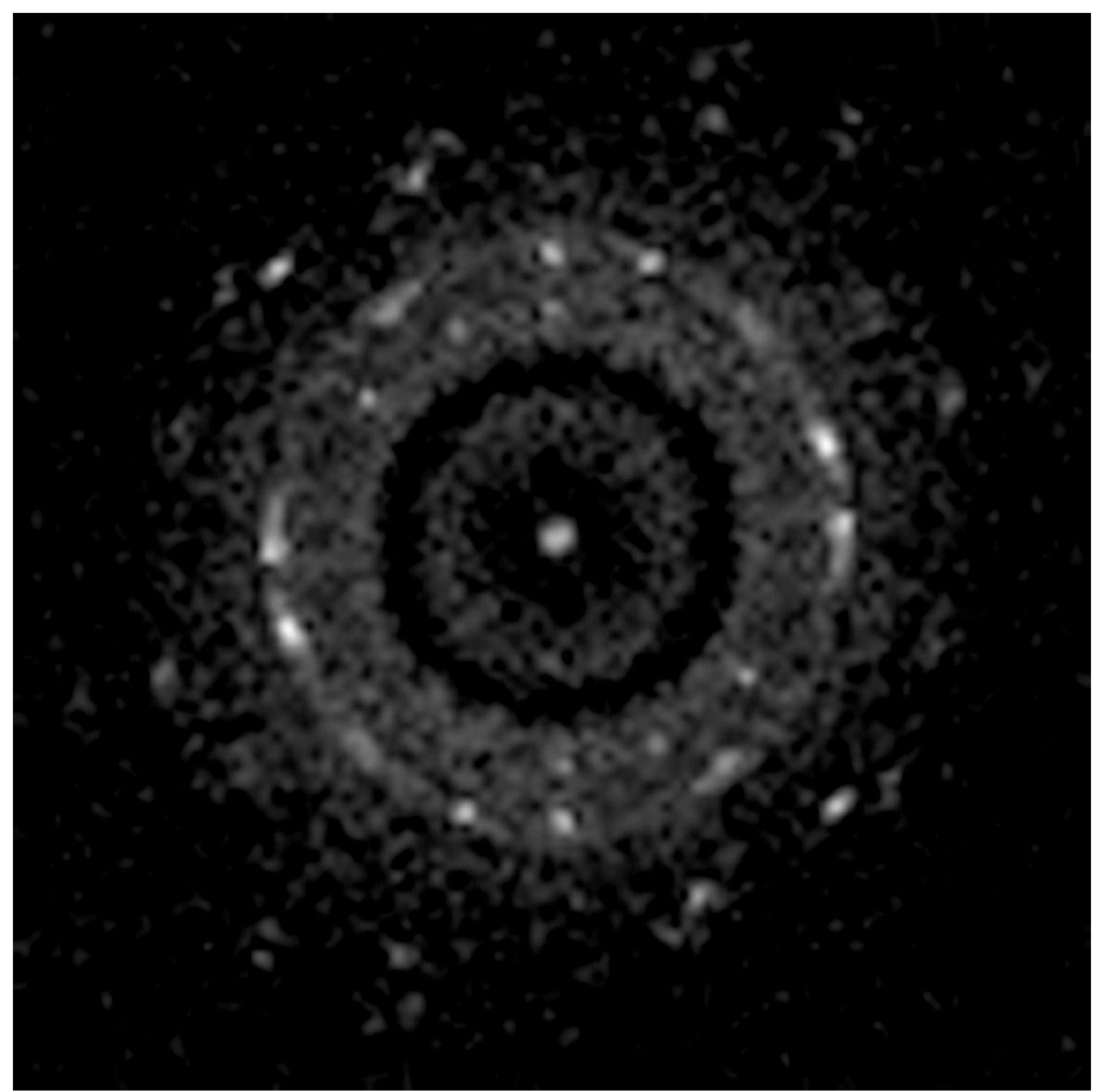

Figure 2. The corresponding power spectrum confirms the structure of the crystals are of fcc.

\section{References}

[1] Wuttig M, Yamada N (2007) Phase-change materials for rewriteable data storage. Nat Mater 6(11), 824832.

[2] Dirisaglik F, Bakan G, Jurado Z, Muneer S, Akbulut M, Rarey J, Sullivan L, Wennberg M, King A, Zhang L (2015) High speed, high temperature electrical characterization of phase change materials: metastable phases, crystallization dynamics, and resistance drift. Nanoscale 7(40), 16625-16630.

[3] Noe P, Vallee C, Hippert F, Fillot F, Raty J-Y (2017) Phase-change materials for non-volatile memory devices: from technological challenges to materials science issues. Semicond Sci Technol 33(1), 013002.

[4] Kooi BJ, Groot WMG, De Hosson JTM (2004) In situ transmission electron microscopy study of the crystallization of $\mathrm{Ge}_{2} \mathrm{Sb}_{2} \mathrm{Te}_{5}$. J Appl Phys 95(3), 924-932.

[5] Carter CB, Williams DB (Eds.) (2016) Transmission electron microscopy: diffraction, imaging and spectrometry, Springer, Berlin Heidelberg, 17-80 